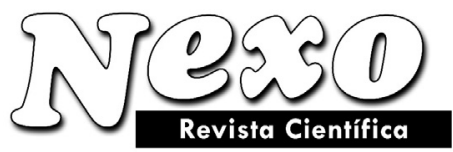

\title{
Indicadores para la evaluación de la calidad ambiental del hábitat urbano
}

\author{
A. Zúñiga* \\ Programa de Estudios Ambientales Urbanos y Territoriales, Universidad Nacional de Ingeniería \\ PO Box 3214, Managua5, Nicaragua \\ e-mail: zunigaaa00@gmail.com
}

(recibido/received: 12-May-2009; aceptado/accepted: 14-Julio-2009)

\begin{abstract}
RESUMEN
La presente investigación consistió en el diseño y aplicación de una metodología para la evaluación de la calidad del medio construido en asentamientos humanos urbanos. El proceso metodológico inició con el establecimiento del estado del arte sobre la materia, destacando los aspectos conceptuales sobre la sustentabilidad como máxima aspiración a lograr en la evaluación de un asentamiento y determinando los componentes significativos de la problemática objeto de trabajo. Posteriormente se caracterizó el sistema vigente de asentamientos urbanos en Nicaragua y se determinaron los asentamientos objeto de estudio. Se identificaron las clasificaciones tipológicas existentes sobre los asentamientos humanos urbanos nicaragüenses, las cuales se completaron mediante la propuesta de una nueva forma de agrupación según la estructuración de los componentes, lo que permitió la selección de los asentamientos donde se aplicó el instrumental. A continuación se desarrolló la metodología de evaluación conformado por el instrumental teórico, los histogramas de evaluación ambiental y las matrices de evaluación de la calidad. Se finalizó el proceso de validación del instrumental diseñado, aplicándose a cuatro asentamientos seleccionados, con base en una muestra del 5\% del total de asentamientos de la zona de estudio elegida. Se valoraron los resultados obtenidos, relacionándolos con otros análisis del área, lo que posibilitó demostrar su efectividad y eficacia.
\end{abstract}

Palabras claves: asentamientos humanos urbanos; hábitat urbano; medio construido; calidad ambiental.

\begin{abstract}
This research involved the design and implementation of a methodology for assessing the quality of the built environment in urban settlements. The methodology began with the establishment of the state of the art on the subject, emphasizing the conceptual aspects of sustainability as the highest aspiration to achieve in the evaluation of a settlement and determining the significant components of the problem to work. Subsequently characterized the existing system of urban settlements in Nicaragua and identified settlements under study. Typological classifications were identified existing urban human settlements in Nicaragua, which was completed by proposing a new way of grouping according to the structuring of components, allowing the selection of settlements where you applied the instrumental. The following evaluation methodology developed consists of the theoretical tools, the histograms of environmental assessment and parent assessment of quality. It ended the instrumental validation process designed to apply to four selected settlements, based on a sample of $5 \%$ of all settlements in the area of study selected. We evaluated the results, relating them to other area analysis, which enabled to demonstrate their effectiveness and efficiency.
\end{abstract}

Keywords: urban human settlements, habitat urban built environment, environmental quality.

* Autor para la correspondencia 


\section{INTRODUCCIÓN}

El modelo de desarrollo económico, los conflictos políticos y bélicos, la fuerte migración rural y las secuelas de desastres naturales de todo tipo aún no superados, explican en parte la problemática que Nicaragua enfrenta en materia de vivienda y que repercute directamente en las condiciones de salud de su población. Este déficit se ha visto agravado por el marcado deterioro de las capas populares y el impacto negativo en la infraestructura productiva y vial, provocada por el huracán Félix en Septiembre de 2007.

Después de los años 90, uno de los problemas que enfrentaron los asentamientos en general, era el alto volumen de población que habitaba en el campo o la ciudad en viviendas o fincas asignadas por programas de reforma agraria rural o urbana sin contar con alguna garantía legal. Esta situación generó inseguridad por demandas de antiguos dueños y la imposibilidad de conseguir préstamos con bajas condiciones hipotecarias seguras, debido a la incertidumbre legal en que permanecía la propiedad.

En Centroamérica, Nicaragua ha compartido con Honduras los niveles más bajos de desarrollo económico. Los conflictos militares y políticos en los que se ha visto envuelta a lo largo de los últimos 20 años han repercutido seriamente en su economía, por lo que se clasifica en el segundo lugar de los países más pobres de América. Según el İndice de Desarrollo Humano del PNUD (1998) Nicaragua ocupa el lugar número 126, mientras que Honduras se ubica en el sitio 119 y Costa Rica en la posición 34.

El desarrollo de asentamientos humanos en Nicaragua, está fuertemente condicionado, en primer lugar, por la construcción de viviendas y por ende, por la capacidad económica de sus moradores; en segundo lugar por las tendencias urbanas y rurales y finalmente por pautas que sigue la población en algunas regiones del país. Una de las causas del fracaso de los asentamientos urbanos es la falta de planificación y de instrumentos que permitan valorar las condiciones del hábitat. Los problemas del ambiente son identificados como los desequilibrios en los ecosistemas, facilitando que se identifique como sinónimo de ambiental todo aquello que se refiera a lo natural, lo verde y la magnitud de los efectos que sobre el equilibrio del planeta ha provocado el uso de los recursos naturales; esto se refleja en el marco legal e institucional vigente en el país, el cual está siendo transformado a través de un proceso de descentralización de la gestión ambiental auspiciado por el Gobierno Central por medio del ente rector de los recursos naturales, que es el Ministerio del Ambiente y los Recursos Naturales (MARENA).

Actualmente se discute ampliamente la problemática ambiental en diversos sectores y su reflexión lentamente está derivando en la consideración de que existe un problema ambiental urbano de una magnitud considerable, cambiando la visión exclusiva de lo ruralista de la problemática ambiental.

El deterioro físico, espacial, social y económico de las ciudades se configura como un problema comparable y estrechamente relacionado con los grandes problemas ambientales. La modernidad de las ciudades nicaragüenses, el deterioro de los recursos naturales y la contradicción entre ciudad y campo, exigen día a día una solución urgente y profunda, lo que ha implicado en la toma de medidas necesarias que permitan preservar los recursos naturales y promover una política de reutilización, conservación y mantenimiento. Se requiere de una acción social de concientización que establezca una mejor relación hombre-naturaleza, ciudad-campo, medio construido-medio natural, con el ser humano $y$ sus diferentes actividades de incorporación en la sociedad.

En este marco era necesario y urgente desarrollar una investigación ambiental que facilitara métodos de planeación de las ciudades y los asentamientos humanos urbanos y que introdujera la concepción ambiental como elemento estructurante de un desarrollo integral que involucrara la visión interdisciplinaria que interviniera estrechamente en todo el fenómeno ambiental de los asentamientos humanos; para evitar la irregularidad ambiental que sufre actualmente el territorio, como producto de su situación al margen de las disposiciones legales y normativas.

\section{METODOLOGÍA}

El proceso metodológico empleado en el diseño de la investigación se dividió en dos etapas; la primera donde se definió el tema y el objeto de estudio, y la segunda donde se precisaron los objetivos y se definió la hipótesis. La primera etapa se constituyó en un momento clave en el trabajo ya que proporcionó la información básica para realizar el análisis posterior, obtenida mediante la aplicación de entrevistas, recopilación de información y análisis y la incorporación de elementos y normas que dieron la pauta para la conformación de un instrumental ambiental de naturaleza integral. 
Cabe destacar que la investigación se desarrolló con apoyo del método dialéctico, yendo de lo general a lo particular y viceversa, proponiendo resultados sobre la base de la combinación balanceada entre la experiencia iterativa, la selección de fuentes de prestigio especializada y los criterios de expertos en el campo de los asentamientos humanos, a fin de cuidar del rigor científico del documento.

En el marco de estos planteamientos metodológicos genéricos, se desarrolló el procedimiento metódicometodológico particular de diseño del instrumental de evaluación de la calidad ambiental del medio construido de los asentamientos humanos en la escala urbana, el cual se englobó en tres grandes momentos:

a) Identificación de variables o componentes ambientales generales que caracterizan a los asentamientos humanos urbanos.

b) Determinación de Indicadores Específicos (IE) para la evaluación ambiental del medio construido de los asentamientos humanos urbanos

c) Definición de las sub variables de cada IE.

Los tres momentos se pueden apreciar en la figura 1.

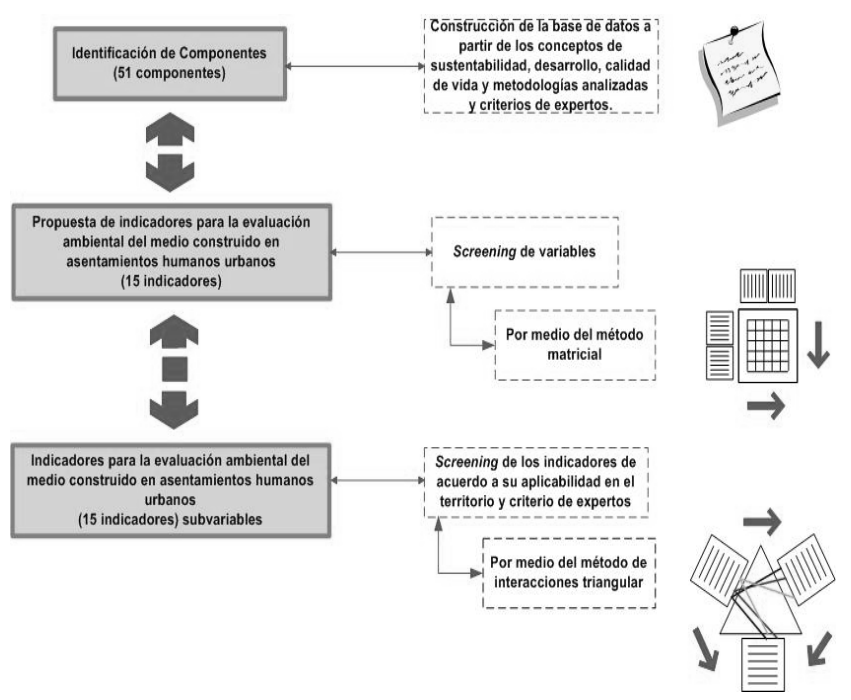

Fig. 1. Proceso metódico-metodológico para la determinación de los indicadores de evaluación. Fuente: A. Zúñiga. 2007

A continuación se describe la secuencia anterior.

Criterios generales y proceso metódico para la determinación de las variables o componentes generales que caracterizan a los asentamientos humanos
El sistema de indicadores se concibió como una guía metodología para evaluar ambientalmente los asentamientos urbanos en el pacífico nicaragüense, e incluyó las siguientes tareas:

- Se balancearon los criterios retomados de prestigiosos científicos e investigadores con amplio reconocimiento mundial en la temática, con los planteamientos de la autora, obtenidos de la realidad nacional, a fin de obtener un instrumental de simple aplicación, pero sumamente efectivo.

- Se asumió lo establecido en el marco legal nacional en relación con los asentamientos humanos urbanos, así como para el municipio de estudio, y los datos de metodologías consultadas que se consideraron aplicables.

- Se aplicaron los aportes de los expertos y actores institucionales y comunitarios, obtenidos de las mesas de transacciones con el comité de expertos y por las discusiones pertinentes en torno a las prioridades locales de los asentamientos urbanos.

- Se asumieron algunos desafíos metodológicos que habían sido sugeridos por Bancrofft (2002), Mingshum (2002), Liepach (2003), Milán (2004) y Gómez Ortega (2005), de donde se destacaron los siguientes:

- Retomar los aportes más recientes de especialistas en asentamientos humanos y las experiencias que hasta la fecha se habían desarrollado a nivel nacional.

- Seleccionar indicadores simples y de fácil aplicación, capaces de identificar y valorar ambientalmente la problemática presente en los asentamientos humanos.

- Limitar, a través del comité de expertos, el número de los indicadores, con la finalidad de garantizar la simplicidad del instrumental.

Como resultado de estos criterios, se identificaron una cantidad grande de componentes o variables generales relacionadas con las características ambientales de los asentamientos urbanos, inferidas de los conceptos de sustentabilidad, desarrollo, calidad de vida y de los soportes teóricos de metodologías estudiadas y de los criterios de expertos.

Una vez identificados los componentes que intervendrían en el proceso de interacción de variables, se procedió a determinar el método por medio del cual se elaboró el procedimiento matricial para la obtención de los indicadores de evaluación. 
Método Matricial (primera aproximación) para la determinación de los indicadores más significativos

La depuración de variables se realizó a través de un proceso de tamizado (screening), mediante el método matricial, para lo cual se consideraron los métodos más actualizados sobre la aplicación de teorías de sistemas para el análisis complejo de factores incidentes $y$ relaciones entre los sistemas humanos y naturales. Estas concepciones se enmarcan en los conceptos contemporáneos e integrales de medio ambiente y desarrollo sustentable, de los cuales a su vez se derivan los de calidad y modo de vida, como paradigmas para el manejo de los asentamientos humanos como expresión del desarrollo social.

Se consultó el mayor espectro posible de actores y personas incidentes en la materia, para obtener la mayor variedad posible de criterios. Este proceso forma parte de los pilares de lo transdisciplinario en el enfoque metodológico.

Los factores, variables y sub variables fueron organizados en "medio construido", "medio natural" y "medio social", tal y como lo hacen muchos actores contemporáneos, permitió acercarse al modelo general de las relaciones entre naturaleza y sociedad.

Método de sistemas de interacción de los factores ambientales: Método triangular (aproximación final)

Una vez establecidos los indicadores, se procedió a aplicar un nuevo proceso de screening, de acuerdo a su aplicabilidad al territorio y en función de los criterios de expertos, lográndose obtener las sub variables de cada indicador.

El tipo de indicador ambiental necesario para evaluar el medio construido en asentamientos humanos urbanos se determinó por la interacción de los factores ambientales entre ellos, por lo que el Método de Sistemas de Interacción de los Factores Ambientales de Velásquez (2006), que parte de una serie de planteamientos básicos y que posteriormente se articulan lógicamente hasta establecer cadenas de interdependencia, fue el más indicado en este caso.

El método ha sido utilizado por dicho autor como herramienta base para la realización de investigaciones académicas y en el desarrollo de trabajos de consulta en el campo del planeamiento ambiental del territorio (ver figura 2).

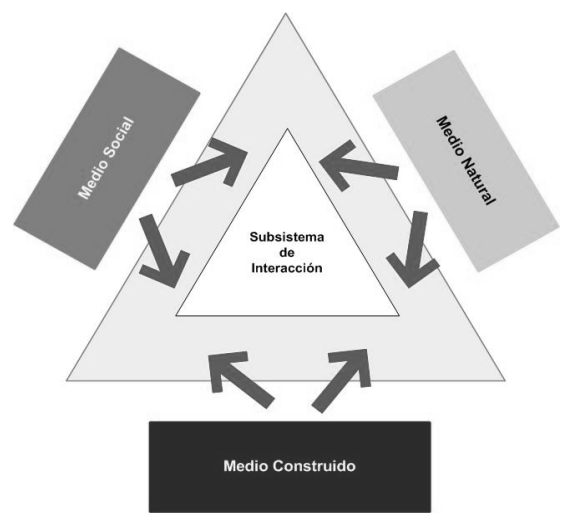

Fig. 2. Proceso metódico triangular. Fuente: A. los 7ínínioa 2007

- ¿Qué factores se relacionan entre sí para evaluar el medio construido en asentamientos humanos urbanos?

- ¿Qué factores se relacionan para modificar la interacción medio construido-medio natural? ¿Qué está pasando con esta interacción?

- ¿Qué relaciones son conflictivas y cuáles son correctas? ¿Cómo se evalúa el medio construido?

- ¿Qué sistema de interacción es el más idóneo para la evaluación?

- ¿Cuál es el indicador que establece el diagnóstico más completo al unir todos los subsistemas?

Estos planteamientos fueron adaptados y adoptados en el en el presente estudio, permitiendo identificar las variables más generales que posibilitaron discriminar las más significativas en el modelo propuesto, tal como se puede apreciar en la columna de la siguiente tabla, denominada "componentes requeridos para medir la calidad ambiental" (ver tabla 1).

Tabla 1. Elementos componentes del análisis de los factores ambientales. Fuente: A. Zúñiga. 2007.

\begin{tabular}{||c||c||c||}
\hline $\begin{array}{c}\text { Factor } \\
\text { Ambiental }\end{array}$ & $\begin{array}{c}\text { Atributos } \\
\text { Relacionados } \\
\text { con el Medio } \\
\text { Construido }\end{array}$ & $\begin{array}{c}\text { Componentes requeridos para medir la } \\
\text { Calidad Ambiental }\end{array}$ \\
\hline \hline \multirow{2}{*}{$\begin{array}{c}\text { Medio } \\
\text { Natural }\end{array}$} & Flora y fauna & Tipología, localización \\
\cline { 2 - 3 } & Suelo & $\begin{array}{l}\text { Temperatura, precipitación, humedad, } \\
\text { vientos }\end{array}$ \\
\cline { 2 - 3 } & Hidrología & Superficial, subterránea \\
\cline { 2 - 3 } & Geomorfología & Geología, morfología, pendientes, altitud \\
\hline
\end{tabular}




\begin{tabular}{|c|c|c|}
\hline $\begin{array}{c}\text { Factor } \\
\text { Ambiental }\end{array}$ & $\begin{array}{l}\text { Atributos } \\
\text { Relacionados } \\
\text { con el Medio } \\
\text { Construido }\end{array}$ & $\begin{array}{c}\text { Componentes requeridos para medir la } \\
\text { Calidad Ambiental }\end{array}$ \\
\hline \multirow{5}{*}{$\begin{array}{l}\text { Medio } \\
\text { Social }\end{array}$} & Paisaje & Paisaje natural, paisaje construido \\
\hline & Modo de vida & Costumbres, tradiciones \\
\hline & Arte & Música, artesanía, arquitectura \\
\hline & $\begin{array}{l}\text { Actividad } \\
\text { económica } \\
\text { productiva }\end{array}$ & $\begin{array}{l}\text { Agrícola, construcción, comercio y } \\
\text { artesanía; vestuario y calzado }\end{array}$ \\
\hline & Población & $\begin{array}{l}\text { Población económicamente activa, } \\
\text { población económicamente inactiva }\end{array}$ \\
\hline \multirow{5}{*}{$\begin{array}{c}\text { Medio } \\
\text { Construido }\end{array}$} & $\begin{array}{l}\text { Estructura } \\
\text { urbana }\end{array}$ & $\begin{array}{l}\text { Configuración } \text { urbana, } \\
\text { urbana, uso de suelo, potencialidada } \\
\text { turística, estratificación social, marco } \\
\text { legal }\end{array}$ \\
\hline & Vivienda & $\begin{array}{l}\text { Densidad habitacional, funcionalidad, } \\
\text { tipología, características constructivas }\end{array}$ \\
\hline & Edificaciones & $\begin{array}{l}\text { Funcionalidad, tipología, características } \\
\text { constructivas }\end{array}$ \\
\hline & Equipamiento & $\begin{array}{l}\text { Red vial, transporte, educación, salud, } \\
\text { parques y deportes, servicios municipales }\end{array}$ \\
\hline & Infraestructura & $\begin{array}{l}\text { Agua potable, drenaje sanitario, drenaje } \\
\text { pluvial, energía, telecomunicaciones }\end{array}$ \\
\hline
\end{tabular}

Determinación de los subsistemas de interacciones de los factores ambientales para la evaluación del medio construido

Para establecer los subsistemas de interacción, se relacionaron aquellos atributos que permitieran evaluar diferentes situaciones en el marco cotidiano de los habitantes de los asentamientos urbanos, y se analizaron las interacciones del medio construido con dichos asentamientos..

Al relacionar los atributos, se obtuvieron una serie de subsistemas de interacciones, los cuales generaron a su vez indicadores ambientales. Cada subsistema dio como resultado un indicador que posee un alcance determinado, cumple objetivos específicos y tiene su propio sistema de medición y representación por medio de diagramas. A estos productos se les denomina Indicadores Específicos (IE).

Para una clara comprensión del sistema de interacciones, se presenta la nomenclatura para cada uno de los cruces que se realizaron, destacándose en sombreado el subsistema que cruza con los restantes y los resultados que ofrecerán las variables significativas del proceso (ver tabla 2).
Tabla 2. Nomenclatura para la interacción de los factores ambientales. Fuente: A. Zúñiga. 2007.

\begin{tabular}{|c|c|c|c|}
\hline \multirow{2}{*}{$\begin{array}{c}\text { Sistema de } \\
\text { Interacciones } \\
\text { de Factores } \\
\end{array}$} & \multicolumn{3}{|c|}{ Atributos } \\
\hline & $\begin{array}{c}\text { Subsistema } \\
\text { CONSTRUIDO } \\
\end{array}$ & $\begin{array}{c}\text { Subsistema } \\
\text { SOCIAL }\end{array}$ & $\begin{array}{l}\text { Subsistema } \\
\text { NATURAL } \\
\end{array}$ \\
\hline SI - C.1 & - estructura urbana & $\begin{array}{l}\text { - población } \\
\text { - arte } \\
\text { - modo de } \\
\text { vida }\end{array}$ & $\begin{array}{l}\text { - } \text { suelo } \\
\text { - geomorfología }\end{array}$ \\
\hline $\mathrm{SI}-\mathrm{C} .2$ & - vivienda & $\begin{array}{l}\text { - población } \\
\text { - actividad } \\
\text { económica } \\
\text { - productiva } \\
\text { - arte } \\
\text { - modo de } \\
\text { vida }\end{array}$ & $\begin{array}{l}\text { - paisaje } \\
\text { - clima } \\
\text { - suelo } \\
\text { - geomorfología }\end{array}$ \\
\hline $\mathrm{SI}-\mathrm{C} .3$ & - edificaciones & $\begin{array}{l}\text { - población } \\
\text { - actividad } \\
\text { económica } \\
\text { productiva } \\
\text { - arte } \\
\end{array}$ & - suelo \\
\hline $\mathrm{SI}-\mathrm{C} .4$ & - equipamiento & $\begin{array}{l}\text { - actividad } \\
\text { económica } \\
\text { productiva }\end{array}$ & $\begin{array}{l}\text { - paisaje } \\
\text { - flora } \\
\text { - fauna } \\
\text { - suelo } \\
\text { - hidrología } \\
\text { - geología } \\
\end{array}$ \\
\hline SI - C. 5 & - Infraestructura & $\begin{array}{l}\text { - población } \\
\text { - actividad } \\
\text { económica } \\
\text { productiva }\end{array}$ & $\begin{array}{l}\text { - paisaje } \\
\text { - flora } \\
\text { - fauna } \\
\text { - suelo } \\
\text { - hidrología } \\
\text { - geomorfología }\end{array}$ \\
\hline $\mathrm{SI}-\mathrm{S} .1$ & $\begin{array}{l}\text { - estructura urbana } \\
\text { - equipamiento }\end{array}$ & $\begin{array}{r}\text { - paisaje } \\
\text { urbano }\end{array}$ & $\begin{array}{l}\text { - flora } \\
\text { - fauna } \\
\text { - clima } \\
\text { - suelo } \\
\end{array}$ \\
\hline $\mathrm{SI}-\mathrm{S} .2$ & $\begin{array}{l}\text { - } \text { estructura urbana } \\
\text { - vivienda } \\
\text { - equipamiento }\end{array}$ & $\begin{array}{l}\text { - modo de } \\
\text { vida }\end{array}$ & $\begin{array}{l}\text { - clima } \\
\text { - geomorfología }\end{array}$ \\
\hline $\mathrm{SI}-\mathrm{S} .3$ & $\begin{array}{l}\text { - estructura urbana } \\
\text { - equipamiento }\end{array}$ & - arte & - suelo \\
\hline $\mathrm{SI}-\mathrm{S} .4$ & $\begin{array}{l}\text { - } \text { estructura urbana } \\
\text { - vivienda } \\
\text { - edificaciones }\end{array}$ & $\begin{array}{l}\text { actividad } \\
\text { económica } \\
\text { productiva }\end{array}$ & $\begin{array}{l}\text { - flora } \\
\text { - fauna } \\
\text { - suelo } \\
\text { - geomorfología }\end{array}$ \\
\hline $\mathrm{SI}-\mathrm{S} .5$ & $\begin{array}{l}\text { - estructura urbana } \\
\text { - vivienda }\end{array}$ & •población & - suelo \\
\hline $\mathrm{SI}-\mathrm{N} .1$ & $\begin{array}{l}\text { - } \text { estructura urbana } \\
\text { - vivienda, } \\
\text { - edificaciones } \\
\text { - equipamiento, } \\
\text { - infraestructura } \\
\end{array}$ & $\begin{array}{l}\text { - paisaje } \\
\text { urbano }\end{array}$ & - flora \\
\hline $\mathrm{SI}-\mathrm{N} .2$ & $\begin{array}{l}\text { - estructura urbana, } \\
\text { - equipamiento } \\
\text { - infraestructura }\end{array}$ & $\begin{array}{l}\text { - paisaje } \\
\text { - actividad } \\
\text { económica } \\
\text { productiva }\end{array}$ & - fauna \\
\hline $\mathrm{SI}-\mathrm{N} .3$ & $\begin{array}{l}\text { - } \text { estructura urbana } \\
\text { - vivienda } \\
\text { - edificaciones } \\
\end{array}$ & $\begin{array}{l}\text { - paisaje } \\
\text { urbano }\end{array}$ & - clima \\
\hline $\mathrm{SI}-\mathrm{N} .4$ & $\begin{array}{l}\text { - } \text { estructura urbana } \\
\text { - vivienda } \\
\text { - Edificaciones } \\
\text { - Equipamiento } \\
\text { - infraestructura }\end{array}$ & $\begin{array}{l}\text { - paisaje } \\
\text { - arte } \\
\text { - actividad } \\
\text { económica } \\
\text { productiva } \\
\text { - población }\end{array}$ & - suelo \\
\hline $\mathrm{SI}-\mathrm{N} .5$ & $\begin{array}{l}\text { - vivienda } \\
\text { - edificaciones } \\
\text { - equipamiento } \\
\text { - infraestructura }\end{array}$ & $\begin{array}{l}\text { - población } \\
\text { - actividad } \\
\text { económica } \\
\text { productiva } \\
\text { - arte } \\
\end{array}$ & - hidrología, \\
\hline
\end{tabular}


Proceso para la obtención de indicadores cualitativos para la evaluación de la calidad ambiental del hábitat urbano

El proceso mediante el cual se diseñaron los indicadores incluyó la elaboración de una propuesta de variables o componentes generales, que posteriormente permitió la selección de las variables más significativas (tamizado de variables) a través del método matricial y la interacción sistémica de las variables que intervienen en el sistema ambiental (medio construido-medio socialmedio natural) mediante el uso del método triangular, lo que permitió obtener los Indicadores Ambientales Específicos.

Finalmente, se agruparon los Indicadores Específicos, a través de la asociación por afinidad en relación con sus características o atributos, conformando los Indicadores Generales (IG), tal como se puede apreciar en la figura 3.

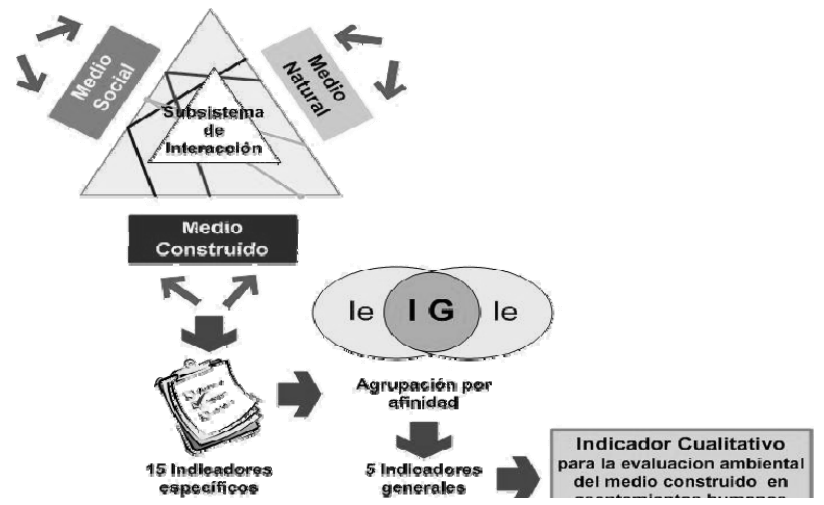

Fig. 3. Interacción sistémica de variables para la obtención de Indicadores Específicos. Fuente: A. Zúñiga. 2007

\section{Proceso de selección de variables más significativas}

Como se ha mencionado, la definición de las variables más significativas que posibilitaron obtener los indicadores de evaluación de la calidad del medio construido de los asentamientos humanos urbanos, se logró una vez identificadas las variables generales, las cuales fueron cruzadas mediante un proceso que permitió la interacción entre ellas. Para ello se utilizó el sistema de interacciones de los factores ambientales, por medio del cual se relacionaron sus atributos, de manera que se obtuvieron subsistemas de interacciones que fueron definidos mediante una matriz de sistemas con base en un rango o Escala de Valoración. A los indicadores específicos se les aplicó la fórmula matemática (1) que permitió calcular el indicador total resultante del medio construido o calidad ambiental del medio construido:
$\mathrm{E}=\Sigma \mathrm{EP} / \Sigma \mathrm{P}$

Donde para cada indicador:

E es la evaluación

$\mathrm{P}$ es el peso o importancia y

EP la evaluación ponderada

La determinación de la escala de evaluación, fue definida mediante el siguiente proceso:

- Determinación del diseño de los parámetros de evaluación cualitativa de cada indicador.

- Representación de las evaluaciones cualitativas por medio de histogramas y de las evaluaciones cuantitativas por matrices síntesis. Juntas conforman el instrumental metodológico.

\section{Proceso de evaluación}

A partir de este proceso se propusieron las diferentes situaciones evaluativas que fueron ajustadas en cuanto a la asignación del puntaje en función de los criterios de especialistas, actores y de los resultados de la aplicación del instrumental, estableciéndose rangos más cerrados en las condiciones de excelencia y más amplios en las peores condiciones; por entenderse que bastaría una situación negativa cuya insatisfacción paralizaría el funcionamiento del sistema y lo afectaría severamente.

Se asumió una Escala de Evaluación para medir la calidad ambiental del medio construido que es directamente proporcional al nivel de la calidad de vida existente en el asentamiento humano urbano. El estado del indicador se valora con mayor puntaje en la medida en que su calidad de vida sea mejor, y se asigna un menor puntaje al Indicador cuando sucede lo contrario (ver tabla 3):

Tabla 3. Escala de Evaluación para los indicadores de la calidad ambiental del medio construido.

\begin{tabular}{|c|c|c|c|c|c|}
\hline Escala & \multirow{2}{*}{ Situaciones } & \multicolumn{3}{|c|}{ Valoración } & \multirow{2}{*}{ Recomendación } \\
\hline $\begin{array}{c}/ \\
\text { Rango) } \\
\end{array}$ & & Color & Descripción & Significado & \\
\hline 5 & \multirow[t]{2}{*}{$\begin{array}{l}\text { Mas favorables } \\
\text { o excelentes. }\end{array}$} & & & & $\begin{array}{lr}\text { Monitorear } & \text { el } \\
\text { asentamiento } & \text { en }\end{array}$ \\
\hline $5-4,7$ & & & $\begin{array}{c}\text { Verde } \\
\text { intenso }\end{array}$ & Muy Bien & $\begin{array}{l}\text { base a los } \\
\text { lineamientos } \\
\text { planteados en el } \\
\text { instrumental. }\end{array}$ \\
\hline 4 & $\begin{array}{l}\text { Con algunas } \\
\text { afectaciones o } \\
\text { limitaciones } \\
\text { que afectan su } \\
\text { calidad de vida. }\end{array}$ & O & Verde claro & Bien & $\begin{array}{lr}\text { Analizar } & \text { los } \\
\text { aspectos } & \\
\text { señalados } & \text { para } \\
\text { optimizar } & \text { el } \\
\text { confort del } & \text { sitio } \\
\text { a través } & \text { de } \\
\text { medidas } & \text { de } \\
\text { mitigación. } & \\
\end{array}$ \\
\hline
\end{tabular}




\begin{tabular}{|c|c|c|c|c|c|}
\hline Escala & \multirow{2}{*}{ Situaciones } & \multicolumn{3}{|c|}{ Valoración } & \multirow{2}{*}{ Recomendación } \\
\hline $\begin{array}{c}/ \\
\text { Rango) }\end{array}$ & & Color & Descripción & Significado & \\
\hline $3,89-3$ & $\begin{array}{l}\text { Con } \\
\text { afectaciones } \\
\text { significativas } \\
\text { en algunos de } \\
\text { los } \\
\text { componentes, } \\
\text { que harán de } \\
\text { tomar } \\
\text { medidas que } \\
\text { mitiguen o } \\
\text { corrijan las } \\
\text { situaciones } \\
\text { que degradan } \\
\text { su calidad de } \\
\text { vida. }\end{array}$ & 0 & Amarillo & Regular & $\begin{array}{l}\text { Analizar los } \\
\text { aspectos } \\
\text { señalados para } \\
\text { corregir la } \\
\text { problemática } \\
\text { detectada y } \\
\text { elevar el confort } \\
\text { del sitio a través } \\
\text { de medidas de } \\
\text { mitigación. }\end{array}$ \\
\hline 2 & $\begin{array}{l}\text { De elevada } \\
\text { degradación } \\
\text { de varios de } \\
\text { los } \\
\text { componentes } \\
\text { de s calidad } \\
\text { de vida, con } \\
\text { medidas de } \\
\text { consideración- }\end{array}$ & & Rojo & Mal & $\begin{array}{l}\text { Analizar los } \\
\text { aspectos } \\
\text { señalados para } \\
\text { corregir la } \\
\text { problemática } \\
\text { detectada y } \\
\text { elevar el confort } \\
\text { del sitio a través } \\
\text { de medidas que } \\
\text { permitan darle } \\
\text { calidad de vida a } \\
\text { la población. }\end{array}$ \\
\hline
\end{tabular}

Asimismo, se aplica a la escala de Evaluación el "semáforo ambiental", artificio gráfico de uso muy común en el campo de la evaluación ambiental, donde a las situaciones menos favorables se les asigna un color rojo o amarillo, y a las más favorables o mejores se les asigna el color verde, suministrando visualmente una mejor precepción del estado del indicador.

\section{RESULTADOS Y DISCUSIÓN}

El primer resultado obtenido en la presente investigación se relaciona con la adopción del planteamiento contemporáneo que define al ambiente como la interacción continua entre el medio social y su medio natural (ver figura 4), superando la visión tradicional que lo entiende desde el punto de vista estrictamente natural.

Siendo la base de este estudio los asentamientos urbanos, y con base en esta definición integral de ambiente, se interpretó que el sistema ambiental debía

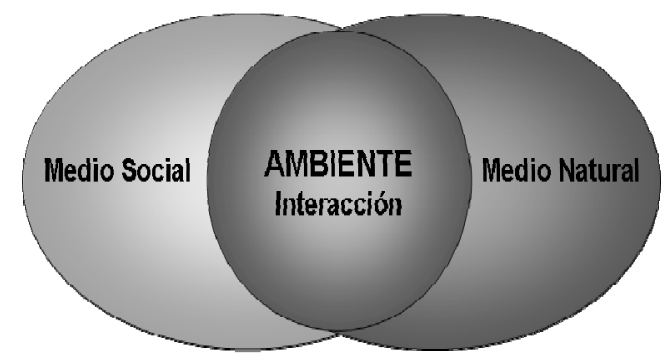

Fig. 4. Interacción del ambiente. A. Zúñiga, 2007 estar conformado por tres medios: el Medio Construido, el Medio Natural y el Medio Social, extrayéndose de este último el medio construido. Esta triada apuesta a la sustentabilidad, en donde se declara que lo deseable es lograr un asentamiento económicamente viable, socialmente justo y ambientalmente sano.

Por lo anterior, las definiciones empleadas en este estudio se encuentran inmersas en los conceptos de ambiente y desarrollo sustentable, de los cuales a su vez se derivan los de calidad y modo de vida como paradigmas para el manejo de los asentamientos humanos como expresión del desarrollo social.

El concepto de desarrollo sustentable se compatibiliza con la protección al medio ambiente, ya que se procura garantizar un crecimiento económico que permita satisfacer las necesidades de la población; en donde se abarquen los aspectos económicos, sociales (incluyendo los aspectos culturales) y ecológicos, adoptando principios que logren un asentamiento económicamente viable, socialmente justo y ambientalmente sano (ver figura 5).

A partir de las reflexiones referidas, sobre temas tan debatidos y abarcadores como los de sustentabilidad,

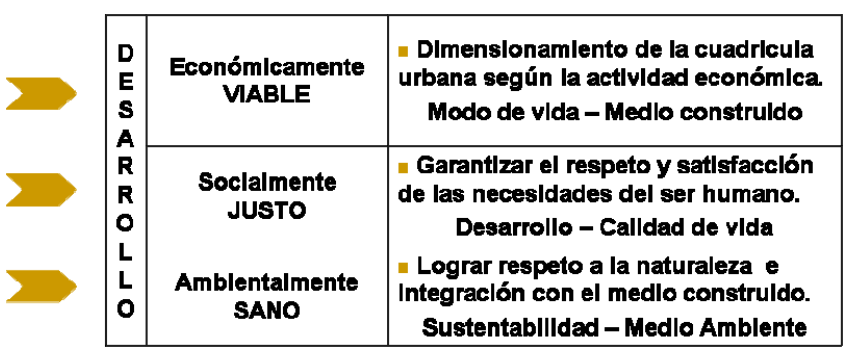

Fig. 5 Principios de Sustentabilidad Asumidos. Fuente: A. Zúñiga, 2007, con base en Gómez Ortega, 2005.

desarrollo y lo urbano, y otros no tan conocidos como calidad de vida; se sintetizaron los aspectos que por su importancia fueron considerados en la determinación del conjunto de indicadores de evaluación ambiental del medio construido en asentamientos humanos en la escala urbana.

Al considerar dichos aspectos en el diseño de los indicadores se permitió evaluar asentamientos existentes o bien permitir el desarrollo de nuevos asentamientos, considerando criterios de sustentabilidad en estrecho 
diálogo con el territorio, garantizando la satisfacción y expectativas del poblador urbano.

Como resultado de los aportes de los especialistas en asentamientos humanos, experiencias nacionales en la materia, las inferencias de los conceptos contemporáneos de ambiente, sustentabilidad, desarrollo y calidad de vida y los soportes teóricos de metodologías estudiadas, se lograron identificar 51 variables generales relacionadas con las características o atributos de los asentamientos humanos urbanos, abarcando la totalidad de los aspectos que tienen que ver con la calidad ambiental de los mismos.

Dado que el estudio de este amplio número de variables complejizaría la evaluación de la calidad del medio construido de los asentamientos urbanos, se limitó la cantidad de componentes para garantizar la simplicidad del instrumental propuesto. Este proceso permitió identificar que el método matricial, sustentado en la valoración de expertos, era el más indicado para reducir el número de variables involucradas y en consecuencia, determinar los indicadores más significativos.

El empleo de un comité de expertos logró incorporarle un enfoque transdisciplinario a la investigación, ya que la cantidad y diversidad de actores considerados representaron tanto a disciplinas científicas como a las áreas de la vida cotidiana en los asentamientos humanos.

Para esta investigación se retomó el planteamiento de que el medio construido de un asentamiento urbano dialoga con lo natural y lo social, por lo que no sólo establece vínculos con la sociedad sino con la cultura como medio de desarrollo del modo de vida de esa misma sociedad y que a su vez es complementado por los aspectos económicos de ese entorno. Aunque el medio construido es parte del medio social, su estudio por separado permitió diferenciar lo super estructural (cultura, sociedad, estética, economía, etc.) de lo físico o construido.

Todo lo que no es natural pertenece al campo de lo creado por el ser humano y por lo tanto es un producto social. Este producto social se puede dividir inicialmente en creación material (medio construido) y medio ideal (como superestructura). Este estudio se enmarcó en el primer tipo, donde para la determinación de variables del medio construido el método aplicado permitió identificar los elementos a considerar en el proceso.

En este marco de discusión, se generó un proceso de decantación de las 51 variables generales a través del método de interacción de factores del medio natural, social y construido (método triangular), lo que permitió establecer 15 subsistemas de interacciones ambientales, los cuales generaron a su vez 15 Indicadores Ambientales Específicos (IE).

La definición de las variables más significativas resultantes de los subsistemas de interacción, posibilitó obtener los indicadores de evaluación específicos de la calidad del medio construido de los asentamientos humanos urbanos. Los Indicadores Específicos permitieron identificar y valorar el estado particular de 15 ámbitos relacionados con el medio construido de los asentamientos urbanos.

Mediante un proceso asociativo de agrupación por afinidades, los 15 Indicadores Específicos generaron 5 categorías de Indicadores Generales (IG), los cuales permitieron identificar y valorar la calidad ambiental del medio construido de los asentamientos urbanos en estudio de forma más integral e integradora (ver tabla 4).

Tabla 4. Indicadores para la evaluación de la calidad ambiental del hábitat urbano. Fuente: A. Zúñiga. 2007

\begin{tabular}{|c|c|c|c|}
\hline & \multicolumn{3}{|c|}{ Variables significativas } \\
\hline & $\begin{array}{l}\text { Indicadores } \\
\text { Generales }\end{array}$ & & Indicadores Específicos \\
\hline \multirow{3}{*}{1} & \multirow{3}{*}{$\begin{array}{l}\text { Convivencia } \\
\text { habitacional } \\
\text { (Comunidad) }\end{array}$} & 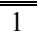 & Calidad estética de los asentamientos \\
\hline & & 2 & Accesibilidad a una vivienda confortable. \\
\hline & & 3 & Cohabitabilidad espacial \\
\hline \multirow{3}{*}{2} & \multirow{3}{*}{$\begin{array}{l}\text { Configuración } \\
\text { del hábitat }\end{array}$} & 4 & Cobertura espacial del equipamiento \\
\hline & & 5 & Funcionalidad de las redes técnicas \\
\hline & & 6 & Calidad del paisaje territorial \\
\hline \multirow{3}{*}{3} & \multirow{3}{*}{$\begin{array}{l}\text { Pertenencia del } \\
\text { hábitat }\end{array}$} & 7 & Confort habitacional \\
\hline & & 8 & Identidad \\
\hline & & 9 & $\begin{array}{l}\text { Condiciones facilitantes para el desarrollo } \\
\text { de actividades económicas - productivas }\end{array}$ \\
\hline \multirow{2}{*}{4} & \multirow{2}{*}{$\begin{array}{l}\text { Regularidad del } \\
\text { hábitat }\end{array}$} & 10 & Orden social \\
\hline & & 11 & Estética urbana \\
\hline \multirow{4}{*}{5} & \multirow{4}{*}{$\begin{array}{l}\text { Adaptabilidad al } \\
\text { medio }\end{array}$} & 12 & Confort climático urbano \\
\hline & & 13 & Adaptabilidad al suelo del hábitat \\
\hline & & 14 & Ordenamiento hidrológico en el hábitat \\
\hline & & 15 & $\begin{array}{l}\text { Niveles de alteración morfológica en el } \\
\text { desarrollo del hábitat }\end{array}$ \\
\hline
\end{tabular}

A su vez, los Indicadores Generales permitieron establecer el Indicador Cualitativo Total de la Calidad 
Ambiental del medio construido de los asentamientos humanos urbanos, que sintetiza el estado final en que se encuentran éstos. Los resultados parciales obtenidos se graficaron y muestran el resultado del indicador total.

\section{CONCLUSIONES}

La base teórica metodológica que soporta la investigación fue el resultado de un proceso de adopción y adaptación de conceptos contemporáneos aplicables a la calidad ambiental del medio construido de asentamientos humanos en la escala urbana, en donde la autora toma partido por una definición de ambiente donde se articula lo social, lo construido y lo natural.

En consecuencia, el instrumental de evaluación diseñado se basa en un abordaje equilibrado de los conceptos de sustentabilidad y calidad de vida, y consideró los principales aportes y limitaciones provenientes de los modelos e indicadores existentes, lo que unido a la toma de criterios y al consenso de especialistas y de actores estratégicos, posibilitó la elaboración de una metodología novedosa basada en indicadores de evaluación específicos a las condiciones de Nicaragua.

Se caracterizó el Sistema de Asentamientos Humanos vigente en Nicaragua, lo que permitió inferir que dicha clasificación no incluye el estudio del medio construido de los mismos, y por lo tanto, no existen estudios sobre la calidad ambiental de éstos.

Al aplicar y valorar el instrumental en los asentamientos urbanos de diferentes tipologías seleccionadas, se pudo demostrar la efectividad de este instrumental para la evaluación de la calidad ambiental del medio construido, a partir de la propuesta de indicadores simples.

Esta metodología constituye un procedimiento que contribuye al propósito de darle cuerpo científicotécnico al proceso de evaluación de la calidad ambiental en Nicaragua, con énfasis en los asentamientos urbanos de los territorios en estudio, posibilitando conocer y actuar sobre la problemática que afecta el ambiente construido nacional, con posibilidades de generalizar los resultados obtenidos.

\section{REFERENCIAS}

Gómez, G (2005). Instrumental para la evaluación de los componentes del medio construido que influyen en la calidad de vida en asentamientos rurales concentrados (ARC) de Santiago de Cuba. Tesis para optar por el título de doctor en ciencias técnicas, Tutor: Dr. Arq. Rubén Bancrofft Hernández.

Gómez, G. (2005). Calidad de Vida. Curso de Calidad de Vida de la Especialidad en Vivienda Social y Calidad de Vida. UNI / PEAUT.

Gómez, G. (2005). Calidad de Vida. Curso de Medio Construido y Calidad de Vida. Doctorado en Ciencias del Ambiente. UNI / PEAUT.

Gómez, R. (2000) OPS. Salud en la Vivienda en los países que conforman la Red Interamericana de Centros de Salud en la Vivienda. El Caso de Nicaragua.

González, D. (2005). Medio Ambiente Construido y Desarrollo Sustentable. Facultad Arquitectura ISPJAE. La Habana, Cuba.

MARENA (1996). Ley 217: Ley General del Medio Ambiente y los Recursos Naturales. Gaceta No. 105.

Mendoza, F. (2006) Metodología para el Estudio del Medio Ambiente. PEAUT / UNI. Nicaragua.

PNUD (1998). Informe sobre Desarrollo Humano en Nicaragua.

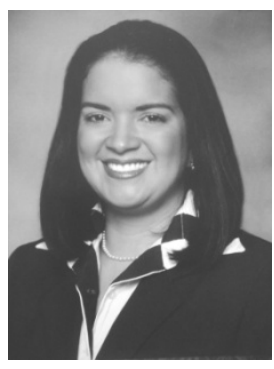

Alicia Alejandra Zúñiga Portillo se graduó de Arquitecta en la Universidad Nacional de Ingeniería (UNI) en 1998. Realizó estudios de especialidad en Vivienda Social y Calidad de Vida y de maestría en Medio Ambiente y Desarrollo Urbano y Territorial en el Programa de Estudios Ambientales Urbanos y Territoriales (PEAUT-UNI). Candidata a Doctor en Ciencias del Ambiente. Su área de investigación es el medio ambiente con énfasis en asentamientos humanos. 